\title{
NIRS0S: Observations of early-type galaxy secular evolution spanning the $\mathrm{Sa} / \mathrm{S0} /$ disky-E boundaries
}

\author{
Eija Laurikainen $^{1}$, Heikki Salo ${ }^{2}$, Ronald Buta ${ }^{3}$ and Johan Knapen ${ }^{4}$ \\ ${ }^{1}$ Finnish Centre for Astronomy with ESO (FINCA), Univ. of Oulu/Turku \\ email: eija.laurikainen@oulu.fi \\ ${ }^{2}$ Dept. of Physics, Univ. of Oulu, ${ }^{3}$ Dept. of Physics and Astronomy, Univ. of Alabama, \\ ${ }^{4}$ Instituto de Astrofísica de Canarias, Dept. de Astrofísica, Univ. de La Laguna
}

\begin{abstract}
NIRS0S (Near-IR S0 galaxy Survey), is a $K$-band survey of $\sim 200$ early-type disk galaxies, mainly S0s, 2-3 mag deeper than the 2Micron All Sky Survey. In depth morphological analysis was done, in which multi-component structural decompositions played an important role. Possible implications to internal dynamical galaxy evolution were discussed. S0s were suggested to be former spirals in which star formation has ceased, forming a parallel sequence with spirals (see Fig. 1). If that evolution is faster among the brighter galaxies, the observed magnitude difference between the barred and non-barred S0s could be understood. Bars are suggested to play a critical role in such evolution. For example, the inner lenses in the bright non-barred S0s can be explained as former barlenses (inner parts of bars), in which the elongated bar component has dissolved. We suggest that the last destructive merger event happened at a fairly large redshift.
\end{abstract}

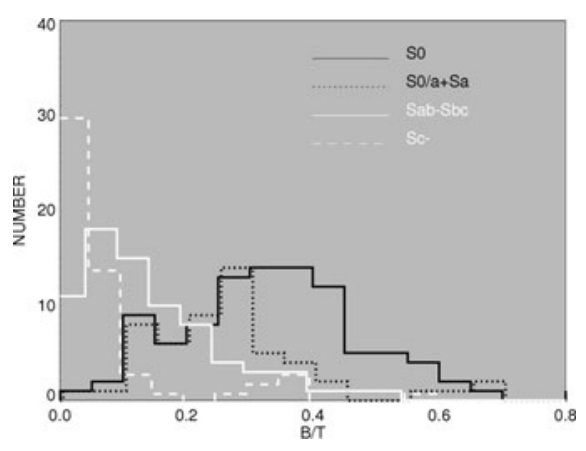

Figure 1. Many S0s have small $B / T$ flux-ratios, overlapping even with the late-type spirals (Laurikainen et al. 2010). This evidence points to a 'parallel sequence', where S0s are spread throughout the Hubble sequence in a similar manner as spirals (S0a, S0b, S0c, see van den Bergh 1976; Cappellari et al. 2011; Kormendy \& Bender 2012).

\section{References}

Cappellari, M. et al. 2011, MNRAS 4161680

Kormendy, J. \& Bender, R. 2012, ApJS 198, 2

Laurikainen, E. et al. 2010, MNRAS 405, 1089

van den Bergh, S. 1976, ApJ 206, 883 